\title{
A Multi Views Approach for Remote Sensing Fusion Based on Spectral, Spatial and Temporal Information
}

\author{
FARAH Imed Riadh \\ ${ }^{1}$ National school of computer science, Manouba University, Laboratory RIAD-GDL, \\ 2Telecom-Bretagne, Department ITI, Brest, \\ 1Tunisia \\ ${ }^{2}$ France
}

\section{Introduction}

Nowadays, operational earth observation satellites provide a large variety of multi-sensor, multi-temporal and multi-modal data. Signals generated by miscellaneous sensors needs to be sampled, filtered, fused, stored, and interpreted (Yu \& Christakos, 2010). Each of these data-processing steps must be conducted in an efficient way to conserve data fidelity. Amidst these research areas, remote sensing community is notably interested in studying multi-source images fusion issues. Over last past decades, information fusion has emerged to manage large amounts of multi-source data in the military field (Mahler, 2007). Recently, substantial amount of research has been dedicated to data fusion techniques development and adaptation for signal and images processing applications. Therefore, data fusion is now largely adopted in several fields including, but are not limited to, satellite and aerial imaging, medical imaging, sonar and radar, robotics, etc. (Stathaki, 2008).

Until recently, images fusion has become a worthy tool in remote sensing image processing and received great attention for satellite image interpretation. Motivations for images fusion are numerous and predominantly justified by application issue (Farah et al., 2008b). Fusion techniques aim to produce an enhanced single view with extended information content by combining intelligently multi-modality data coming from different sources. However, remote sensing images are characterized by their unique spectral, spatial, temporal and directional dimensions depending fundamentally on the nature of the corresponding sensor (Farah et al., 2010). Thus, image fusion can be looked with different points of view; each one is designed to answer specific research requirements and to meet a particular need.

Typically, for an efficient fusion, some questions must been answered before deciding about the fusion approach: What is the objective of image fusion? Which types of data are the most useful? What is the most "appropriate" method of fusion to achieve study goals? What technique is used for results assessment? (Pohl \& Van Genderen, 1998).

Moreover, numerous challenging research issues are related to developing new approaches for remotely sensed signals managing and interpretation. In most actual researches, sensors must operate in an unfriendly environment with many complications. Therefore, an image processing method must be able to deal effectively with limited resources and 
missing/noisy data (Yu \& Christakos, 2010). Imperfections are inherent in all applications fields and arise from measurements errors, spatio-temporal variability and numerical approximation, etc. Therefore, the fusion procedure is associated, generally, with the calculation of uncertainties. In our case, we use the term imperfection to denote limitations associated with data. Here, we refer to one or more of the characteristics: imprecise, uncertain, incomplete, inconsistent and vague when using this term. In a remotely sensed context, we identified the following types of imperfection:

- Imperfections related to nature: it is a consequence of the spatio-temporal variability of the natural phenomena (precipitation, climate changes, etc.) which introduces a random function into the physical process.

- Imperfections related to data: most researchers agree that it is impossible to identify the variability and the local data complexity through some points of measurement.

- Uncertainty related to model parameters: influenced by data imprecision.

Difficulties in fusion process lie also with the problems of redundant information reducing and the large volume data managing. In addition, data specially extracted from each individual source are naturally incomplete (Farah et al., 2003). Hence, developing an efficient data fusion technique must take into account these factors. Some requirements to the images processing algorithms included:

- Tolerance to noise, un-calibrated data frequently associated with remote sensing data.

- Resource constrained computation.

- Robustness and reliability: if any data sources are missing

- Ambiguity reducing.

Having answered these requirements and questions, appropriate technique for data fusion may be chosen. Conjointly, specific data features must been taken into account at all fusion process stages. These features differ from one area to another typically including heterogeneous, large amount, and multi-objective data. Improving knowledge and providing a better description of the real world is the major goal of information fusion techniques. To achieve this ambition, remotely sensed images must be mapped to semantic level for data analysis, interpretation, and decision making (Bentabet et al., 2002). Such mapping requires further efforts and effective images processing tools (Gamba et al., 2005). This chapter focuses on image fusion techniques for remotely sensed applications. Designing a fusion process requires a good assimilation of techniques foundations, a welldefined input data as well as an effective assessment metrics. The objectives of this chapter are to contribute to the apprehension of image fusion approaches including concepts definition, techniques ethics and results assessment. It is structured in five sections. Following this introduction, a definition of image fusion provides involved fundamental concepts. Respectively, we explain cases in which image fusion might be useful. Most existing techniques and architectures are reviewed and classified in the third section. In fourth section, we focuses heavily on algorithms based on multi-views approach, we compares and analyses the process model and algorithms including advantages, limitations and applicability of each view. The last part of the chapter summarized the benefits and limitations of a multi-view approach image fusion; it gives some recommendations on the effectiveness and the performance of these methods. These recommendations, based on a comprehensive study and meaningful quantitative metrics, evaluate various proposed views by applying them to various environmental applications with different remotely sensed images coming from different sensors. In the concluding section, we fence the chapter with a summary and recommendations for future researches. 


\section{Image fusion: definition and fundamentals}

Data fusion is a formal framework defined by means and tools for heterogeneous data alliance (Wald, 1999). Image fusion (IF) has been used in many application areas especially in computer vision and remote sensing fields. Most popular applications concern multisensor fusion combining images from different engines to achieve a high spatial and spectral resolutions. Nowadays, Earth observation satellites provide data covering different portions of the electromagnetic spectrum at different spatial, spectral and temporal resolutions (Hemissi et al. 2009). Multi-source, multi-sensor and multi-temporal data often present complementary information about a surveyed scene, so image fusion appears as an effective way enabling efficient analysis of such data (Farah et al., 2008a). Therefore, data fusion from various sources aids in delineating objects with interest and comprehensive information thanks to complimentary data integration.

The list of image fusion techniques grows as new forms of sensors that are expanded and applied to data acquisition. Many definitions have been proposed from the remote sensing community, where fusion concepts and algorithms have been matured over several decades. Image fusion aims to integrate complementary heterogeneous data and/or multi-view information acquired in several domains. Hence, a multi-view fusion aims to generate an image with higher information degree by considering diverse aspects.

Image fusion means a very wide domain and it is very difficult to provide a precise definition. A number of earlier definitions of sensor, data, images and information fusion have been proposed in the literature (Gamba et al., 2005), among these we can cite:

Def 1: "Fusion ... aims at obtaining information of greater quality; the exact definition of greater quality' will depend upon the application." (Wald, 1999)

Def 2: "...techniques combine data from multiple sensors, and related information from associated databases, to achieve improved accuracy and more specific inferences than what could be achieved by the use of a single sensor alone"(Hall \& Llinas, 1990)

Def 3: “...a multilevel, multifaceted process dealing with the automatic detection, association, correlation, estimation, and combination of data and information from multiple sources" (US Department of Defense)

It was felt in all these definitions that several concepts appear around images fusion. First the term "data" is used in the definition 2, whereas the term "information" is preferred in definitions 1 and 3 . Here, we choose to use the term information in order to designate the whole of what can be fused. Moreover, most definitions treat the term "information" in its entirety. However, several other authors assume that is possible to characterize information into two or three main types (Bloch, 1996). The first type relates to numerical information which may be signal intensity, pixel gray level, etc. The second type is the symbolic information which may be expressed in symbols, proposals (e.g. what is great is not small), rules (e.g. if it's big and it flies, c is a plane), etc. Recently, numerous researches propose a hybrid type of information (Bloch, 1996). We noticed also that the symbolic type has been a little studied in images fusion, although it can be an important source of information. The difficulty lies in formulation of expert knowledge on data and sensors (Stathaki, 2008).

We further denote that all these definitions delineate information fusion as a combination from several sources. So, it is important to clarify the purpose of the term "combination", allowing a new image with more valuable information and which quality cannot be achieved otherwise. Many writers from the computer scientist community understand the 
fusion as the concatenation of multi-sources information. This does not exclude the possibility of obtaining information from a single source after specific treatment. Therefore, the proposal of image fusion is to create new images that are more suitable for further image-processing tasks usually allowing the data amount reducing.

Formally, suppose that we have $m$ sources $S j$ with $j €[1, \ldots, m]$. Each source $S_{j}$ can be characterized by information provided by the $i^{\text {th }}$ source as a function of the observation $X$ noted $s_{j}(X)$. For each observation $X$, these sources should take a decision in a set of $n$ decisions $d_{1}, \ldots, d_{n}$. Each source $S_{j}$ provides information to decision $d_{i}$ about observation $X$ that we denoted $\mathrm{M}_{\mathrm{i}} \mathrm{j}(\mathrm{x})$. Thus the final decision on the observation $\mathrm{x}, \mathrm{E}(\mathrm{x})$ will be taken from the combination of information contained in the matrix $\left(\mathrm{M}_{\mathrm{i}} \mathrm{j}(\mathrm{x})\right)$ given by $(1)$.

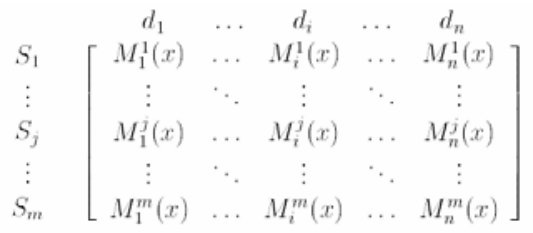

Most of these definitions were focusing too much on fusion techniques despite giving some attention to quality. Once the results of fusion process have been generated, quality evaluation provides convincing indicators about fusion contribution. However, meaning and measurement depend on the particular application. Thus, the effectively evaluation has been a challenging topic among the image fusion community (Gianinetto \& Villa, 2007). Most common image fusion quality evaluation approaches can be classified into two main categories: qualitative approach which considers a visual comparison of results, and quantitative approach involving a set of predefined quality indicators.

\section{Image fusion approaches}

A variety of image fusion schemes have been proposed in the literature, concerning multisources data combination and support decision making. Each fusion method is designed for a specific problem resolution with disparate inputs, processing approach and outputs. This section aims to propose a state of art of images fusion approaches for remotely sensed applications, to study their main ideas and to sort algorithms into respective categories.

\subsection{Data fusion architecture}

Fusion architecture describes how to set and use information sources commonly with mathematical and images processing algorithms in order to perform an efficient fusion operation. Some studies tend to characterize image fusion architecture by data type (Dasarathy, 2001) or by the desired applications (Hall \& Llinas, 1990). In remotely sensed studies, it is more interesting to characterize its structure which can be defined as a fusion cell. Wald (Wald, 1999) structured synthetically the fusion cell into several elementary operations shown in Figure 1.

Information sources, original data or sensors measurements are the main inputs of the fusion cell. Auxiliary information, providing additional data, can be obtained by a specific source processing or deriving out of another fusion operation. External knowledge is designed to support and assist the fusion process by imposing a priori information, which leads us to elect the adequate model for fusion process. In iterative processing, fusion results 
can be used as auxiliary information, since it is not considered as original sources. Finally, it is interesting to get a quality index in addition to results after fusion process. This quality index serves to evaluate the chosen method and to adjust additional information.

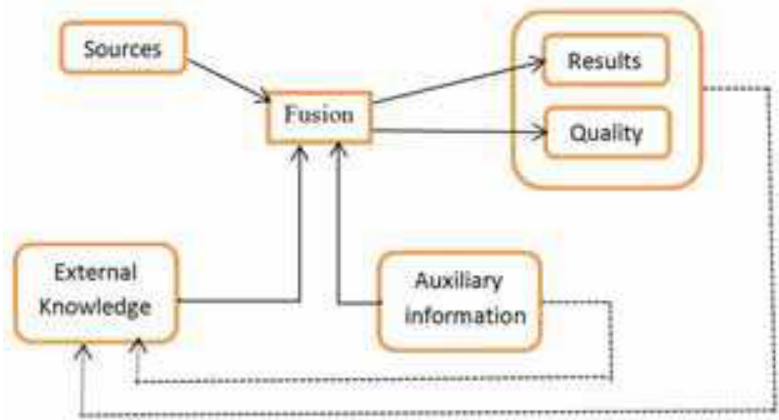

Fig. 1. Formalization of an elementary fusion operation as a fusion cell.

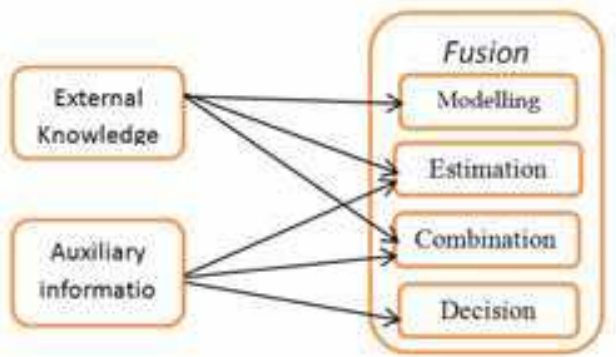

Fig. 2. Fusion process steps.

Three types of architectures are usually considered: centralised, decentralised and hybrid (Lawrence, 2004). The centralised architecture exploits, concurrently or not, input data in a single location. Since this architecture takes into account the whole available sources and knowledge, it provides theoretically an optimal result. Centralised architecture has some drawbacks such as rigidity and noise sensitivity. Therefore, if a particular source has a large error rate, the whole data set is affected which leads to the decrease in the decision quality. Satellite image properties severely limit the use of this type of architecture owing to noise, atmospheric conditions, sensor drifts, etc. Although, decentralised architecture is often adopted since it offers a large flexibility and modularity. Hybrid architectures, which are a combination of centralized and decentralised architectures, may be used recently. According to fusion cell proposed by (Wald, 1999), numerous researches look to the fusion as a compound stage and a succession of several steps (cf. Figure 2); including generally:

- Modelling: the first step of fusion process formulation and it is particularly critical since it tend to choose the fusion formalism (i.e. information representation). It consists generally of determining $\mathrm{M}_{\mathrm{i}} \mathrm{j}$, which can be a distribution, a cost function, etc.

- Estimation: depends on previous step, it is necessary for most fusion formalism since it allows function initialization.

- Combination: The combination step is the heart of fusion operation allowing information consolidation. It meets to choose an appropriate fusion operator 
conforming to the representation formalism defined in step one. Additional information can guide this choice. Most interesting properties of fusion operators are associativity, commutativity, idempotency and adaptability (Bloch, 1996).

- Decision: is final step of fusion operation. Usually, it consists of minimizing or maximizing the combination function. The same function can be also used to calculate a quality index.

\subsection{Image fusion process}

Images fusion techniques are usually conceived following a similar methodology. An overall processing workflow for remotely sensed images fusion is given in figure 3 (Pohl \& Van Genderen, 1998). Later on data collection step, images should be corrected from system errors. Indeed, satellite imagery is influenced by atmosphere during data acquisition and therefore needs some corrections and/or other radiometric enhancements such as edge enhancement. Data are also further radiometrically processed. Following this, data are geometrically corrected due to the height variations in the contained images area.

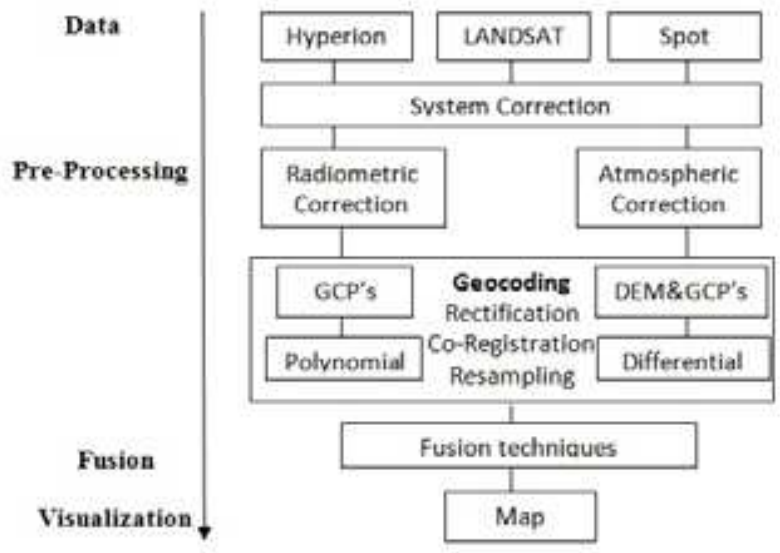

Fig. 3. Flowchart of image fusion process

According to several early studies, fusion techniques are generally grouped into three classes: (1) Colour related techniques, (2) Statistical/numerical methods and (3) combined approaches. The first comprises the colour composition techniques which slice original data into their respective layers, which can be RGB, IHS, HSV or more luminance-chrominance. Statistical approaches use a mathematical approach for data integration. They involve addition, multiplication, differencing and rationing treatments. Combined approaches involve integration of both statistical as well as colour related techniques (Mahler, 2007).

Otherwise, some other researches tend to classify techniques depending to their fusion level. It is often written that fusion takes place at three levels in data fusion: pixel, feature (attribute) and decision. In pixel-based fusion, the information associated with each pixel is obtained by fusing the set of corresponding pixels in source images. In the feature-level approach, each sensor generates a feature vector for a specific object in the scene, which are then fused. In the decision-level fusion, each sensor performs independent processing scheme, and then outputs from each sensor are thereafter combined via a fusion process. 
Techniques referring to feature and decision level are generally deriving from a large range of areas including pattern recognition, artificial learning, artificial intelligence, etc.

Until recently, fusion levels are also discussed in their terminology and their number (Gamba et al, 2205). In several studies four analysis levels are preferred: symbolic, feature, pixel and signal level. The goal of the signal-based fusion is to improve the signal-to-noise ratio.

We can notice that there is confusion between information type and fusion level. Hence signal level can be considered as the pixel level for remote sensing applications. In addition, despite the laborious development of sensors, most images have a low spatial resolution. Recent researches (Farah et al., 2010) suggest analysing remote sensing data at sub-pixel level. Thus, we update in this chapter images fusion techniques classification by adding the sub-pixel level to standard above pixel, feature and decision levels. This new classification is summarized by figure 4 . Figure 5 shows the various fusion inputs/outputs, to which we added the ability to have entrances at different levels. This figure is an illustrative example of all cases that we can meet by adding the sub-pixel level. We recall here that the fusion process can play the role of selection, transformation, extraction, and information classification i from multiple sources.

In the following sections, we propose to illustrate some fusion level by proposing a specific view. For each of them, we present application schema, used data and obtained results.
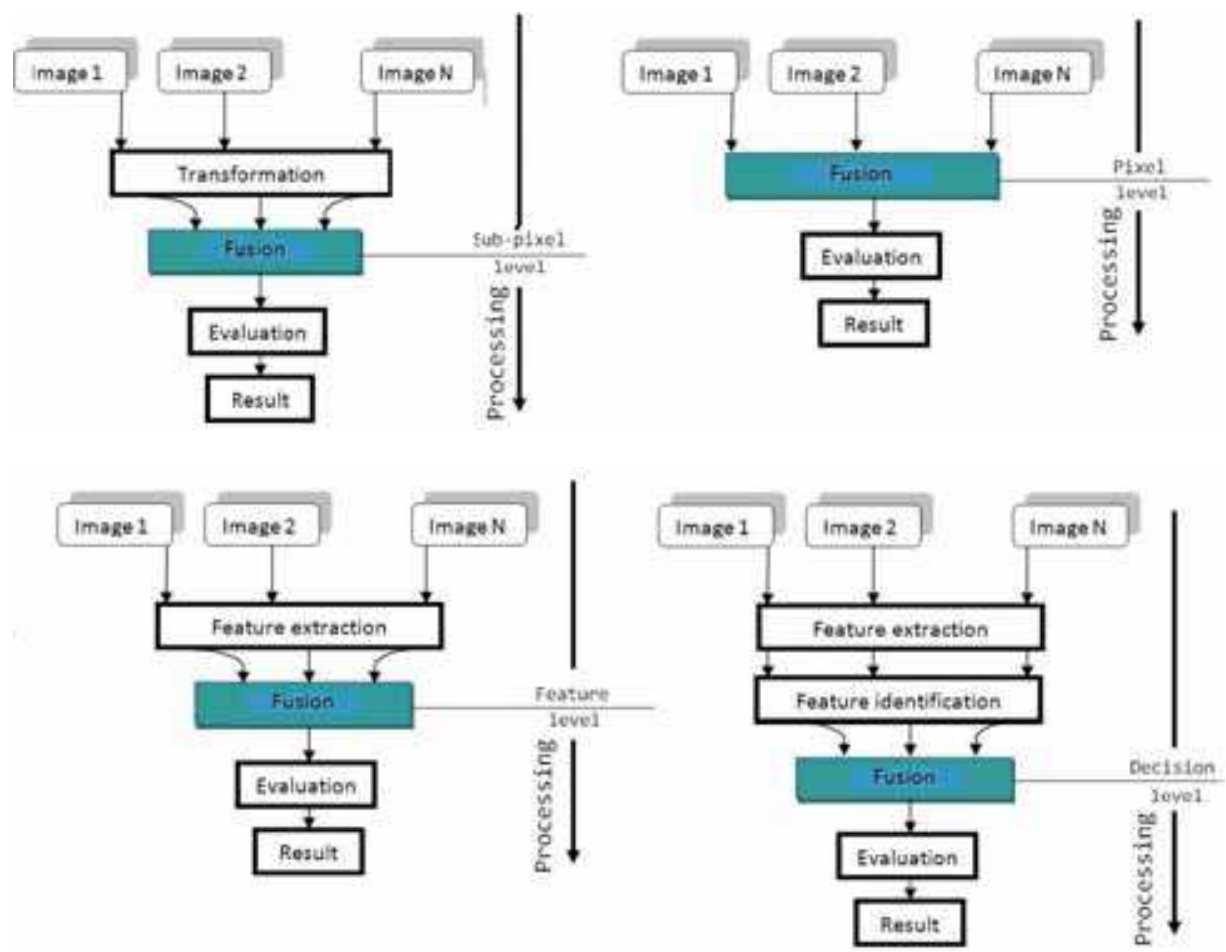

Fig. 4. Proposed classification of fusion techniques 


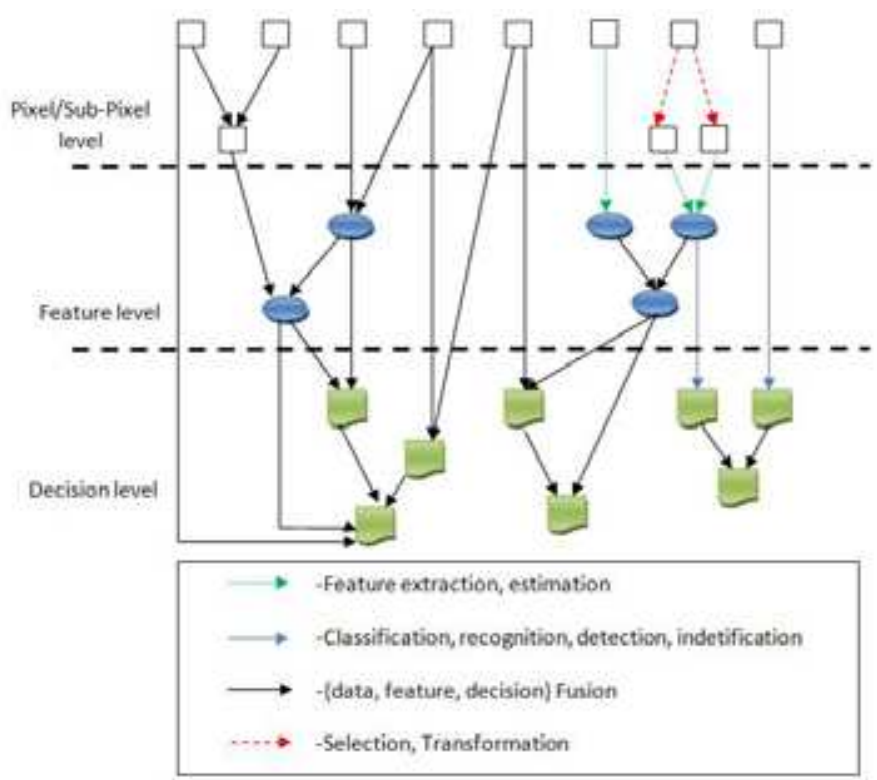

Fig. 5. Inputs/outputs of fusion process

\begin{tabular}{|c|c|}
\hline Sub-pixel level & $\begin{array}{l}\text { Specral unmixing, Mathematical morphology, Second } \\
\text { Order Statistics, Iterative Back-Projection, Wavelet } \\
\text { decomposition, Markov chain/MRF, etc. }\end{array}$ \\
\hline Pixel level & $\begin{array}{l}\text { Neural, fuzzy, neuro fuzzy approaches } \\
\text { Voting Strategies, Wavelets, Regression fusion, Filters, } \\
\text { Colour Normalized transformation, etc. }\end{array}$ \\
\hline Feature level & $\begin{array}{l}\text { Cluster Analysis, Neural Networks } \\
\text { Bayesian Inference, Evidential Fusion } \\
\text { Expert Systems, Logical Templates, etc. }\end{array}$ \\
\hline Decision level & $\begin{array}{l}\text { Classical Inference, Bayesian Inference } \\
\text { Evidential fusion, Contextual Fusion } \\
\text { Voting Strategies, Expert Systems } \\
\text { Neural Networks, Fuzzy Logic } \\
\text { Blackboard Syntactic Fusion, etc. }\end{array}$ \\
\hline
\end{tabular}

Table 1. Fusion approaches review depending on fusion level

\section{Towards a multi-view approach of satellite images fusion}

To overcome problems arising satellite images fusion, we propose a new mufti-view approach intended to enhance images fusion and interpretation. It is designed with diverse fusion schemes and dealing with multi-sources, multi-sensor data and symbolic information. Based on the fact that a unique fusion scheme is impossible to achieve today, we present in this chapter an approach declined on several multiform views. So, fusion practitioners and readers can easily adopt one of these views related to their own problems 
and application areas. Our contribution lies on a novel conception of fusion process offering more flexibility and providing a largest adaptation aptitude. In fact, the proposed approach is structured under several points of view, each designed to meet a specific need, to solve a peculiar problem. The first view tries to overcome the difficulties related to the presence of mixed pixels by performing a sub-pixel probability fusion. The purpose of the second view will be to fuse information extracted from the image with symbolic knowledge in the subpixel level. The last view aims to resolve the conflict related to choice of the optimal fusion technique by combining optimally several approaches. In the following sections, we outline in detail each point of view by emphasizing on its application criteria, proposed fusion process and outputs.

\subsection{View 1: Towards an intelligent Sub-pixel multi-sensor satellite image fusion 4.1.1 Introduction}

Recently, with the development of miscellaneous satellite sensors, a wide variety of remotely sensed data have become available for scientific studies. As the intensity of data acquisition grows, so does the need to combine multi-sensor images in order to extract the most useful information. However, most studies tend often to fuse multi-sensor images by combining straightly radiometric pixels values. This assumption suffers from pixels heterogeneity due to the low spatial resolution of most satellite images (figure6-a). In this view, we introduce a new multi-sensor fusion approach for land cover classification. The proposed approach is an exhibition of multi-sensor images fusion in the presence of mixed pixels considering that the fusion is performed in the sub-pixel level.
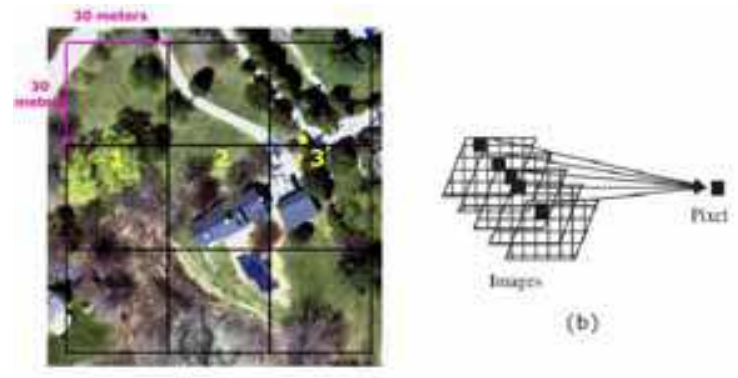

(b)

(a)

Fig. 6. (a): Satellite images heterogeneity, (b): mixed pixel representation

\subsubsection{Proposed approach}

The considered approach focuses on multi-sensor images fusion for land cover recognition. Outlined method is applied to both optical and radar images considering that each sensor is associated with a well-defined spectral band. If optical images are easier to interpret, SAR images are very interesting for land cover studies since they are not bound to the daylight constraint and cloudless conditions, allowing an image acquisition independently of weather conditions (Pohl \& Van Genderen, 1998). Therefore, considering the well-known advantages and disadvantages of each sensor, it seems logical to combine optical and SAR data for an enhanced apprehension of land cover types.

The proposed approach includes various stages for multi-sensors images processing and fusion. Generic flowchart is summarized by figure7. After data collection and pre- 
processing, the proposed approach begins by extract source images thanks to Blind Source Separation methods. Under linearity assumption, the radiometric value of a given pixel can be seen as a mixture of physically independent sources (Farah et al., 2003) (c.f figure 6-b). Thereafter, we generate a set of source images and source signals, each outlining a specific land cover type. Extracted sources evaluation is performed in the next step, allowing additional knowledge discovering from most informative sources signals. To further improve images interpretation, the framework promises a source knowledge representation capabilities delineated as a set of decision rules. Hence, multi-source information fusion produce a valuable understanding of the observed site by decreasing the uncertainty related to single sources (Mansour et al., 2000). In our study, we assumed that multi-sensor adopted images have negligible registration problems, which implies that the objects in all images are geometrically aligned (Goshtasby, 2005). In the following sub-sections, we describe this knowledge representation, as well as the components of the architecture and the interpretation steps.

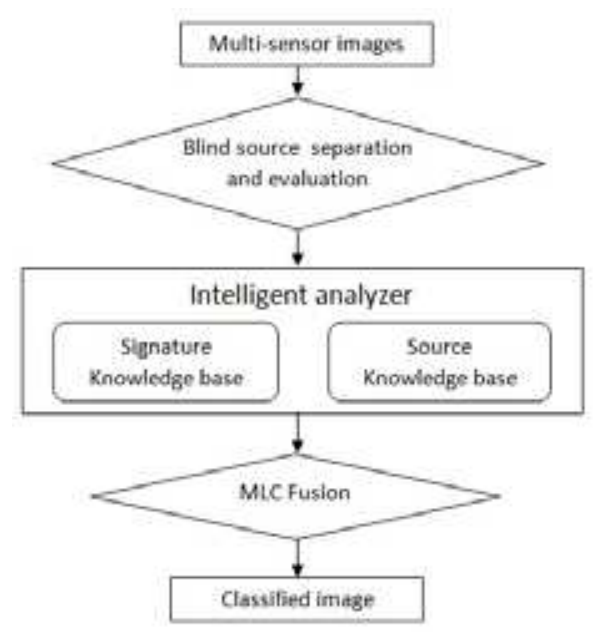

Fig. 7. Workflow of proposed approach

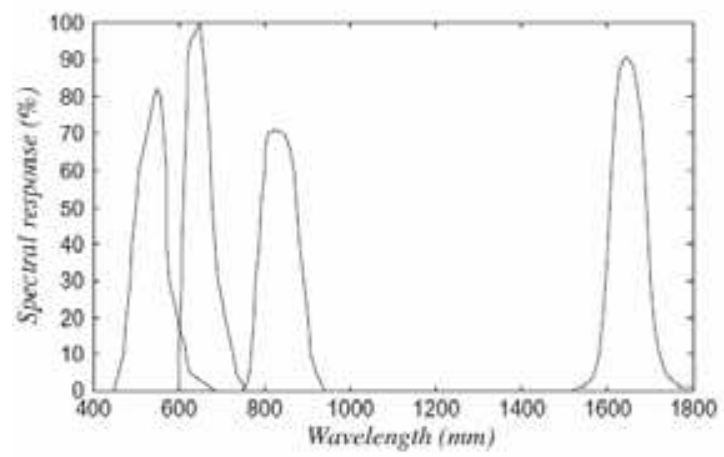

Fig. 8. Typical spectral sensitivity of SPOT4. 


\subsubsection{Blind source separation}

The BSS problem aims to retrieve unknown original signals from their mixtures (measured signals). Its main assumptions are the mutual independence and non-Gaussianity of sources (Mansour et al., 2000). Thus, we attempt to separate observed signals into a set of other signals, such that the regularity of each resulting signal is maximized, and the regularity between the signals is minimized (i.e. statistical independence is maximized). If we admit the linearity of mixing process, the model of BSS can be expressed by:

$$
X=A \times S+N
$$

where $X$ is an $n \times p$ observed image matrix; each of its rows determines the reflectance of the observed image according to a given spectral band. $S$ is an $m \times p$ source images matrix; each of its rows determines the reflectance of one source image. A is an $n \times m$ mixing matrix; each of its columns is called the directional vector associated to the corresponding source. $\mathrm{N}$ is defined as an $n \times p$ matrix realized from a spatially additive white Gaussian noise considered as negligible.

Many approximate methods have been proposed in order to solve equation (2) (Cao \& Liu, 1996). The adapted algorithms in our approach are approximate diagonalization of eigenmatrix (JADE-2D) (Cardoso \& Souloumiac, 1993), second order blind identification (SOBI2D) (Belouchrani, 1997) and fast-independent component analysis (Fast-ICA-2D) (Hyvärinen \& Oja, 1997) algorithms. Source separation can be obtained by optimizing a contrast function that can be based on entropy, mutual independency, higher order statistics, etc. Each of these algorithms takes as an input a matrix $X$ representing the set of multi-sensor images. The goal of all these BSS algorithms is to solve equation (2), in which A (mixing matrix) and S (source images) are the unknown components. After source images extraction, we propose to evaluate their information content using the following criteria, which help us to select just the most informative sources to the fusion process.

The entropy source: This criterion can be interpreted as the degree of information granted by each source image. We use the entropy source in order to assort source images and electing those having a maximum of information degree. Entropy source criterion is given by:

$$
E(S)=-\sum_{n} P_{S}(n) \log _{2} p_{s}(n)
$$

where $S$ and $p_{s}(n)$ denote respectively the source image and the probability of gray level value $n$ of $S$.

Source mutual information (SMI): To evaluate the performances of BSS algorithms, we use the SMI criterion in order to quantify the separation rate between extracted sources. It's based on the concept of mutual information (Zadeh \& Jutten 2005) and defined as:

$$
\begin{aligned}
& E\left(S_{1}\right)=-\sum_{n} P_{s 1}(n) \log _{2} p_{s 1}(n) \\
& E\left(S_{2}\right)=-\sum_{n} P_{s 2}(n) \log _{2} p_{s 2}(n)
\end{aligned}
$$

where $p_{\mathrm{s} 1}(n)$ and $p_{\mathrm{s} 2}(n)$ are the probability of the pixel value $n$ in sources $S_{1}$ and $S_{2}$, respectively. The entropy of the couple $S_{1}$ and $S_{2}$ is: 


$$
E\left(S_{1}, S_{2}\right)=-\sum_{n 1, n 2} p\left(n_{1}, n_{2}\right) \log _{2} p\left(n_{1}, n_{2}\right)
$$

where $\mathrm{p}\left(\mathrm{n}_{1}, \mathrm{n}_{2}\right)$ is the joint probability of pixel value $\mathrm{n}_{1}$ for $\mathrm{S}_{1}$ and $\mathrm{n}_{2}$ for $\mathrm{S}_{2}$. If the sources are independent, the mutual information of a set of $\mathrm{k}$ sources is defined as follow:

$$
I\left(S_{1}, \ldots, S_{n}\right)=-\sum_{n_{1}, \ldots, n_{n}} p\left(n_{1}, \ldots, n_{n}\right) \log _{2} \frac{p\left(n_{1}, \ldots, n_{n}\right)}{p_{S 1}\left(n_{1} n_{1}\right) \ldots p_{s n}\left(n_{n}\right)}
$$

After entropy and MSI criterion computing, we choose the source images having the maximum of information degree. This will help us to extract a learning area that models the spectral characteristics of each land cover type. The knowledge about the land cover theme will be modelled by an intelligent tool based on decision rules.

\subsubsection{Source signals}

After source images extraction and evaluation, we propose to improve interpretation process by using filters called also sources signals (Farah et al., 2003). Thus, the sensitivity of each source image can be modelled by source filters, which consist of a physical representation of source images sensitivity according to the spectral bands (cf. figure 8).

The sensitivity of multispectral observations according to the wavelength $\lambda$ is represented by $S(k, l, \lambda)$, which can be obtained by sampling and quantifying the spectral sensitivity of optical sensor. Each $i^{\text {th }}$ image for the $(k, l)$ pixel represented by $X_{i}(k, l)$ is observed with a filter of reflectance $R_{i}(\lambda)$. Thus, these images can be written as follows:

$$
X_{i}(k, l)=\int R_{i}(\lambda) S(k, l, \lambda) d \lambda
$$

From equation $(8)$, the $(k, 1)^{\text {th }}$ pixel of the $j^{\text {th }}$ image source $S_{j}(k, 1)$ can be modelled by:

$$
S_{j}(k, l)=\sum_{i} c(i, j) X_{i}(k, l)
$$

where $c(i, j)$ is the unmixing coefficient $\mathrm{A}^{-1}$ of source $\mathrm{j}$ and image $\mathrm{i}$. Combining equations (8) and (9), we obtain:

$$
\begin{gathered}
S_{j}(k, l)=\int U_{i}(\lambda) S(k, l, \lambda) d \lambda \\
\int U_{i}(\lambda)=\sum_{i} e(i, j) \int R_{i}(\lambda)
\end{gathered}
$$

The source images can be regarded as observed images through filters $U_{j}(l)$, called the source signals. Therefore, the sensitivity of each source image extracted from the BSS can be modelled by the source signal.

\subsubsection{Intelligent analyzer}

This module performs the enhancement of source images extracted by multi-sensor BSS module by allowing semantics information assigning and improvement (Cf. Figure 9). For each source image, corresponding source signal will be depicted in terms of source knowledge, offering further information about land cover types. This relation will be expressed by a set of decision rules. 


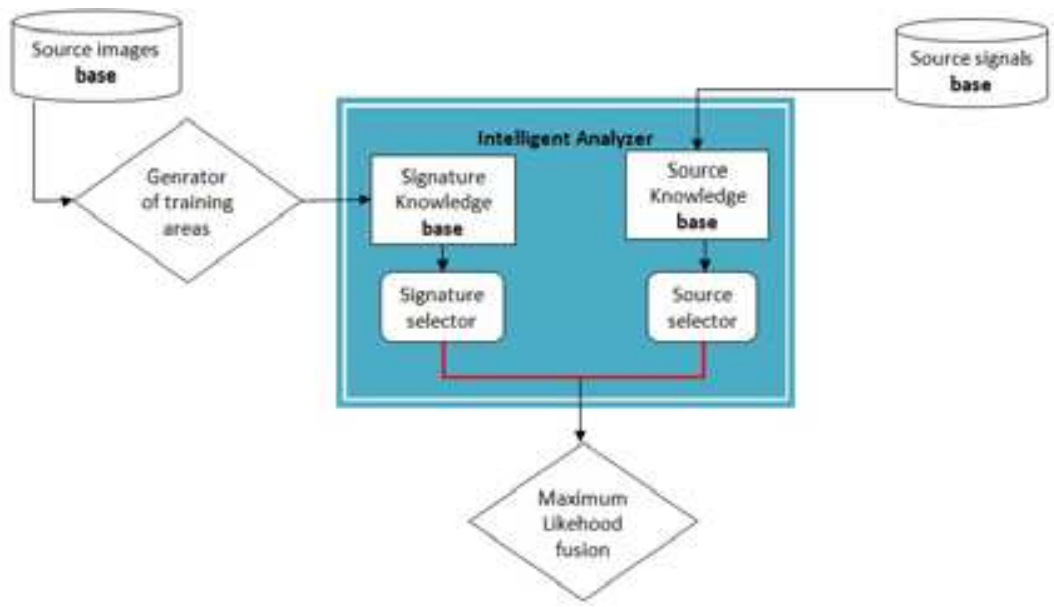

Fig. 9. Workflow of the intelligent analyzer module.

In order to perform a supervised multi-source image fusion, we choose the source image having the maximum information criterion. This operation is carried out by the "source selector" which retains sources with a maximum information degree. This allows us extract a learning area that models the spectral characteristics of each land cover type. The knowledge about the land cover themes will be modelled by an intelligent tool based on decision rules, which will be used by the multi-source image fusion in order to improve and enhance image analysis. Simultaneously, training zones are extracted by the "generator of training areas" module in order to assist satellite image classification and to maximize information extraction. Signatures Knowledge basis concedes semantics to each training zone. The "signature selector" module retains only the one with a maximum percentage of identification. We specify in the following sub-sections each of these sub-modules.

The sources knowledge base. Thanks to this base, land cover type can be recognized for the source images resulting from the multi-sensor BSS step. The base is constructed by using the parameters related to the source signals and the rate of identification of the land cover classes. The facets used to construct this basis are:

a. Parameters

- Interval of $\lambda$ (Interval $\lambda$ ). This parameter represents the wavelength interval corresponding to the maximum value of source signal.

- Maximum value (MaxV). This parameter gives the maximum value of the source signal, indicating that the source is sensitive to a particular type of soil occupation.

b. Decision rules

The production rules are formalized as follows:

$$
\operatorname{If}\left(\operatorname{Max} V\left(S_{i, j}\right)_{i=1: 3, j=1: 5} \succ \sup \left(\operatorname{Max} V\left(S_{k, l}\right)_{k=i, l=1: 5, l \neq j}\right)\right) \& \text { Interval }_{\lambda} \in\left(I_{m}\right)_{m=1: 5} \text { Then }_{-} S_{i, j} i_{i} O_{n}
$$

where $\mathrm{MaxV}$ is the maximum value, $\mathrm{j}$ is the number of the source, $\mathrm{i}$ is the used algorithm $(1=$ Fast-ICA-2D; 2 = JADE-2D; 3 = SOBI-2D), 1 is the number of the source test, and $\mathrm{k}$ is the algorithm used. Im denotes the wavelength interval tests:

The sources selector. This module selects more significant source images depending on their entropy values, sources are ranked in a descending order and significant ones are 
selected. Three sources were chosen from the set of sources affected by radar. From other sources, we choose two sources for each algorithm with maximum entropy criterion which is defined by:

$$
\operatorname{MaxEntropy}\left(\left(S_{i, j, k}\right)_{i=1: 3, j=1: 5, k=0: 1}\right)
$$

where $\mathrm{j}$ represents the number of the source, $\mathrm{i}$ represents the algorithm used $(1=$ Fast- ICA2D; 2 = JADE-2D; $3=$ SOBI-2D), and $k$ indicates whether the source is affected by the radar $(1=$ radar; 0 if not).

The signatures knowledge basis. This knowledge basis is constructed by zones training and from expert knowledge. It includes two main stages:

a. Generator of training zones: this generator extracts a training zone from each source in order to assist the module of fusion and to give an improved classified image. This generation is accomplished by using histogram analysis of each image.

b. Signatures knowledge basis: allows determination of the nature of the training zones extracted from the generator of training zones (GTZ) module.

The multi-source fusion module. This module performs the fusion of selected sources. Maximum likelihood classification (MLC) was used for fusion process. In perform images classification and produce a thematic map.

\subsubsection{Study areas and results}

The proposed method will be illustrated using two different datasets located in central Tunisia. The images come from the ERS2 and SPOT4 satellites. Kairouan, our first selected zone, is situated at approximately $100 \mathrm{~km}$ south of Tunis. Corresponding images for this zone are as follows: (i) a synthetic-aperture radar image from ERS2 acquired on $24^{\text {th }}$ of April 1998, presenting a spatial resolution of $12.5 \mathrm{~m}$, and operating in band C centred on the value frequency $5.36 \mathrm{GHz}$, with a polarization $\mathrm{VV}$ and an incidence angle centred at $26^{\circ}$; and (ii) an optical image of SPOT4 acquired on 31 $1^{\text {st }}$ of May 1998, with a spatial resolution of $20 \times 20 \mathrm{~m}$. The second selected zone is Tunis, centred over the gulf of Tunis. Respective images are as follows: (i) an ERS2 image acquired in June 2003 operating in band C, centred on the value frequency $5.36 \mathrm{GHz}$, with a polarization $\mathrm{VV}$ and an incidence angle centred at $26^{\circ}$; and (ii) an optical SPOT4 image acquired in June 2003, with a spatial resolution of $20 \times 20$.

After data correction and co-registration, blind source module is executed to extract sources images which will be evaluated in the next step. In order to choose the training data, a cartographic map has been used. After having determined the source images and the training and testing zones, we carried out fusion multi-source by MLC for selected zones. The source images used in our experiment are S2 and S4 from Fast- ICA-2D, S1 and S4 from JADE-2D, and S4 and S1 from SOBI-2D. The classification resulting from MLC is an image including five classes related to the various types of land cover (compartmental, humid, urban, lake and vegetation areas (figure 10 (B) (D)).

Confusion matrixes are used for classification evaluation. In order to prove the effectiveness of the proposed method in land cover classification over conventional methods, a thematic map was produced with a maximum likelihood classification (MLC) applied to multispectral imagery without a BSS treatment (Table 2). The overall classification accuracies are listed in tables 2(a) and 2(b), respectively. The improved land-use map is characterized with mixed pixels and more homogeneous regions. The overall accuracy increased considerably from $63 \%$ for MLC applied to multispectral imagery to $85 \%$ with the proposed approach. 

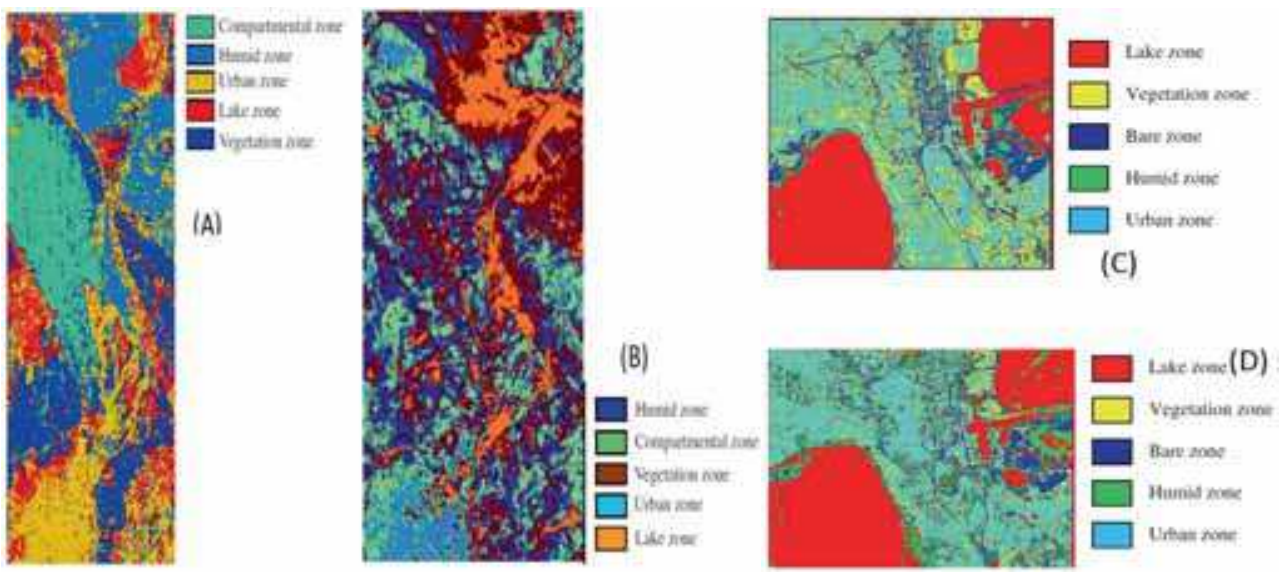

Fig. 10. Classified Kairouan image (A) and Tunis (C) issued from fusion of multi-source images; (B) and (D) are respectively Kairouan and Tunis zone classification with MLC applied to SPOT and ERS imagery.

\begin{tabular}{|c|c|c|}
\hline Class & MLC & Proposed approach \\
\hline Humid & 81.96 & 100.00 \\
\hline Compartmental & 61.76 & 99.44 \\
\hline Vegetation & 58.51 & 99.40 \\
\hline Urban & 52.53 & 97.80 \\
\hline Lake & 75.27 & 95.73 \\
\hline Overall accuracy & 66.01 & 98.47 \\
\hline
\end{tabular}

Table 2(a). Classification accuracy for Kairouan zone,

\begin{tabular}{|c|c|c|}
\hline Class & MLC & Proposed approach \\
\hline Lake & 80.60 & 98.53 \\
\hline Vegetation & 52.98 & 83.93 \\
\hline Bare & 58.31 & 82.67 \\
\hline Humid & 54.04 & 80.76 \\
\hline Urban & 73.88 & 83.80 \\
\hline Overall accuracy & 63.96 & 85.93 \\
\hline
\end{tabular}

Table 2(b). Classification accuracy for Tunis zone.

\subsection{View 2: Towards Neuro-fuzzy approach image fusion}

\subsubsection{Introduction}

Recently, the advent of hyperspectral data provides hundreds of relatively narrow and contiguous bands that may be useful for extracting land-use information. This new form of information can revolutionize the appliance of multisensory images fusion thanks to the wealth of spectral information. Thus, hyperspectral imaging has become a fruitful ally for land cover recognition and natural phenomena monitoring. However, the interpretation of hyperspectral imagery is confronted to several problems such as high data dimensionality, 
spatio-temporal variability of natural phenomena, data imperfection and the requirement of a recurrent expert intervention. So the major dilemma with hyperspectral data interpretation bears upon knowledge integration and fusion flexibility.

As discussed in Section 2, very few studies have focused on an efficient integration of symbolic information in image fusion process. Therefore, we propose in this view a neurofuzzy approach for hyperspectral images interpretation at a sub-pixel level. This investigation serves to consolidate the alliance of the symbolic knowledge into images fusion process and takes advantage of the spectral information provided by hyperspectral imaging. Previous view address spatial and spectral dimensions of images by considering each pixel value as a mixture of several sources. In this view, we show how to analyze also the temporal aspect in satellite images fusion. This investigation is decidedly interesting if information coming from various sensor lack fidelity in the spectral or/and spatial domains.

\subsubsection{Proposed approach}

Our environment is subject to disturbances practiced on variables scales of space and time. On the attempt of natural risk prediction and management, we outline in this view a neurofuzzy fusion strategy for where data fusion is the combination of heterogeneous information from multiple data sources.

The proposed methodology in this view is mainly divided into two stages corresponding to the development of a predictive model of risk hazard monitoring. The first step is "spectral unmixing" allowing abundance maps generation. Each map is relative to a specific endmember in the image. Abundance map of a pure material (source) is a 2D image whose pixel values, ranged between 0 and 1 , indicate the proportion of this material spectrum in each pixel vector. The second step is the fusion of these maps with In situ data using a neuro-fuzzy architecture. The choice of a fuzzy logic has been motivated by data and knowledge imperfection; neural networks have been preferred due to their learning ability allowing model calibration and adaptation (Hemissi et al., 2009).

\subsubsection{Spectral unmixing}

Hyperspectral imaging spectrometers collect images provided by spectral information reflected from surface materials. Each pixel in such image contains a resulting mixed spectrum from reflected sources radiation. Spectral unmixing techniques allow mapping of elements of the scene at the sub pixel level. The objective of this module is to achieve, for each pixel, a reliable extraction of pure spectral signatures and an accurate estimation of their fractional abundances (maps). This investigation should be done using only observed data (hyperspectral pixels), from which the interest of using blind separation of sources techniques, and particularly the independent component analysis (ICA). Formally, the spectral mixture model for a pixel is expressed by equation (2). Then using a BSS technique, mixing proportions of each ground cover material could be retrieved.

In order to obtain abundance maps, we use Independent Component Analysis (ICA) technique which is a blind source separation (BSS) method based on the hypothesis that the independent components (ICs) are statistically independent. Particularly, FAST-2D-ICA (Hyvärinen \& Oja, 1997) algorithm has been adopted to achieve independent components (ICs) generation from hyperspectral images. After ICs computing, we calculate a Priority score for each of them based on higher order statistics (CSOs) (Wang \& Chang, 2006). Since the number of materials in the hyperspectral scene is much less than the dimension of hyperspectral data; we used the Virtual Dimensionality algorithm (Chang, 2004) to estimate 
the number of endmembers in the hyperspectral scene denoted p. We can then classify ICs in order of importance and select only, first $p$ priority ICs. For each of them we elect the pixel with maximum radiometry which may be assumed to be a pure spectral signature (endmember). For endmembers labeling and identification, we use the Spectral Angle Mapper Technical (SAM) (Yuhas, 1992). Outputs of "spectral Unmixing" stage are a set of endmembers and their respective abundance maps. In the next section, we show how to integrate these maps with field (In-situ) data in order to increase fusion and prevision quality.

\subsubsection{Neuro-fuzzy fusion}

This module provides a neuro-fuzzy interpretation of abundance maps generated by BSS analysis. Its main purpose is to build a block of correspondence such as from a set of multisource information (abundance maps and the in Situ data) describing the current situation, it is possible to obtain a prediction of future risks. Fundamentally, the interpretation is essentially seen as a predicting problem by neuro-fuzzy pattern recognition approach.

The use of a neuro-fuzzy model in the problem of hyperspectral images interpretation and for heterogeneous data fusion offers the possibility to model a priori knowledge and linguistic decision rules defined by experts. It also benefits the capabilities and advantages of the fuzzy inference modeled by a parallel neural architecture. Thus, the adjustment of fuzzy system parameters is achieved through neural learning (Lin, 1997). The overall objective of the proposed model is how to associate any new entry to a class of potential risk. For temporal dimension appending, the inputs of our fusion system can also be multitemporal fractions extracted by unmixing a series of hyperspectral images. Therfore, the analysis of these fractions by the neuro-fuzzy model will lead us to analyze change efficentiely by spatial $\backslash$ temporel and spectral consolidation.

Adopted neuro-fuzzy architecture is the FALCON model (Fuzzy Adaptive Learning Control Network) (Lin, 1997), a connectionist model that can be contrasted with a traditional fuzzy logic and decision system into a connectionist structure in terms of its network structure and learning abilities. The FALCON is then a feed-forward multilayer network in which the input nodes represent the input states, the hidden layers work as membership functions and fuzzy logic rules, the output layers represent decision signals. The expert knowledge can be easily incorporated into the model and provides a human understandable meaning to the normal multilayer neural network, the structure avoids the rule-matching time of the inference engine in the traditional fuzzy control system.

The proposed model, shown in Figure 11, consists of five layers. Each node in layer 1 corresponds to one input variable. Each node in layer 2 corresponds to one linguistic label which acts as membership functions representing the terms of the respective linguistic variables. Nodes in layer 3 represent one fuzzy logic rule and perform precondition matching of a rule Layer 3 hence links define the preconditions of the rule. Layer 5 is the output layer. Nodes in layer 4 links define the consequences of rules. The links in layers 2 and 5 are fully connected between linguistic nodes and their corresponding terms nodes. The semantic meaning and function of the neurons are as below:

Layer 1: This layer transfers the input variable to the next layer. Therefore, there are $p+q$ neurons in layer 1 , each represents one input variable. For the $i^{\text {th }}$ neuron in this layer, the input $\left(\mathrm{I}_{\mathrm{i}}{ }^{1}\right)$ and output $\left(\mathrm{O}_{\mathrm{i}}{ }^{1}\right)$ are represented, respectively, as:

$$
I_{i}^{1}=X_{i}^{1} \quad \text { and } \quad O_{i}^{1}=I_{i}^{1}
$$




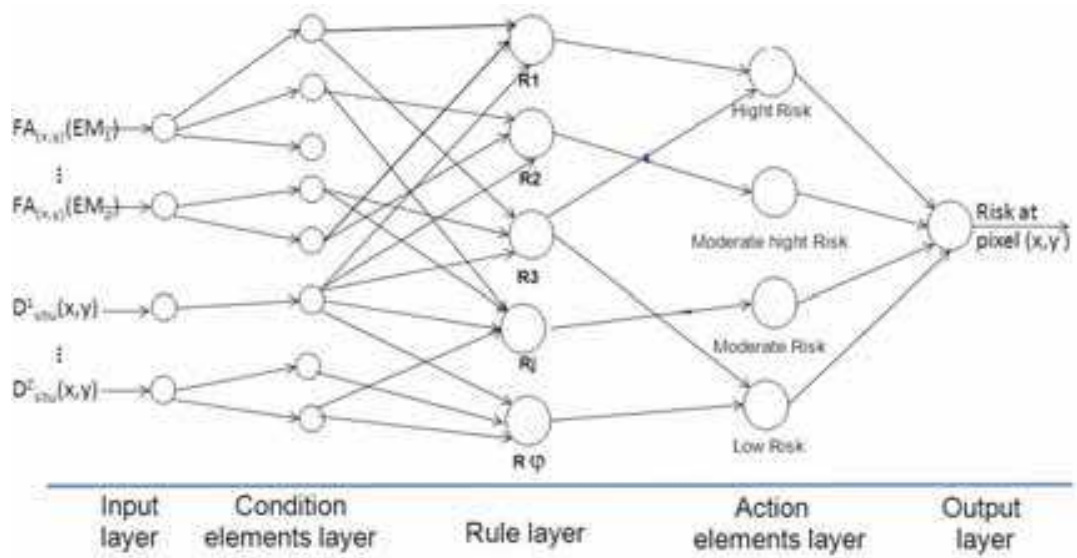

Fig. 11. Proposed neuro-fuzzy model

Where: $\mathrm{FA}_{(x, y)}$ Emp : abundance fraction of endmember $\mathrm{p}$ in pixel with $(\mathrm{x}, \mathrm{y})$ coordinates,

$\left.\mathrm{D}_{(x, y)}\right)^{q}$ : value of the $\mathrm{q}$ In-Situ data, with q: the In-Situ data index

$\phi$ : the rules index

From Eq.(13), the link weight at layer $1\left(\mathrm{~W}_{\left.\mathrm{i}^{(1)}\right)}\right.$ is unity.

Layer 2: Each input feature $x_{i}, i=1,2$ is expressed in terms of membership values, where $i$ corresponds to the input feature and $\mathrm{j}$ corresponds to the number of term sets for the linguistic variable $x_{i}$. We use a single node to perform a bell-shaped membership function Eq.(14):

$$
I_{i j}^{2}=-\frac{\left(O_{i}^{1}-\mu_{i j}\right)^{2}}{2 \sigma_{i j}^{2}} \text { and } O_{i j}^{2}=e^{I_{i j}^{2}}
$$

where $\mu_{\mathrm{ij}}$ and $\sigma_{\mathrm{ij}}$ are, respectively, the center (or mean) and the width (or variance) of the bell-shaped function of the $j^{\text {th }}$ term of the $i^{\text {th }}$ input linguistic variable $x_{i}$. Hence, the link weight at layer $2\left(\mathrm{~W}_{\mathrm{i}}^{(2)}\right)$ can be interpreted as $\mu_{\mathrm{ij}}$.

Layer 3: The links in this layer are used to perform precondition matching of fuzzy logic rules. Hence, the rule nodes perform the fuzzy AND operation:

$$
I_{i}^{3}=\left\{\begin{array}{l}
O_{i j}^{2} \cdots S i \cdots O_{i j}^{2}=\operatorname{Min}\left\{O_{i j}^{2}, \ldots, O_{i j}^{2}\right\} \\
0 \cdots \text { Sinon }
\end{array}\right\} \quad \text { and } O_{i j}^{3}=I_{i j}^{3}
$$

The link weight in layer $3\left(\mathrm{~W}_{\mathrm{i}}(3)\right)$ is then unity.

Layer 4: The nodes in this layer have two transmission modes, i.e., forward and backward. In forward transmission mode, the nodes in this layer perform the fuzzy OR operation to integrate the fired rules which have the same consequence. In the backward transmission mode, the links function exactly same as the layer 2 nodes:

$$
I_{i}^{4}=\left\{\begin{array}{l}
O_{i}^{3} \cdots \operatorname{Si} \cdots O_{i}^{3}=\operatorname{Max}\left\{O_{i}^{3}, \ldots, O_{i}^{3}\right\} \\
0 \cdots \operatorname{Sinon}
\end{array}\right\} \text { and } O_{i}^{4}=I_{i}^{4}
$$

Hence, the link weight $\left(\mathrm{W}_{\mathrm{i}}^{(4)}\right)=1$. 
Layer 5: The nodes of the layer 5 links attached to them act as the defuzzifier. If $\mu_{\mathrm{ij}}$ and $\sigma_{\mathrm{ij}}$ are, respectively, the center and the width of the membership function of the $j^{\text {th }}$ term of the its output linguistic variable, then the Eq.(17) can be used to simulate the center of area defuzzification method:

$$
I_{i}^{5}=\sum_{i} W_{i}^{5} O_{i}^{4} \text { and } O_{i}^{5}=I_{i}^{5}
$$

Here the link weight in layer $5\left(\mathrm{~W}_{\mathrm{i}}{ }^{(5)}\right)$ is $\mu_{\mathrm{ij}} \mathrm{O}_{\mathrm{ij}}$.

Based on this connectionist structure, a supervised gradient-descent learning procedure is developed to determine the proper centers $\left(\mu_{\mathrm{ij}}\right)$ and widths $\left(\sigma_{\mathrm{ij}}\right)$ of the term nodes in layers 2 and 4. To set up the neuro fuzzy model, a hybrid learning algorithm from a set of supervised training data was developed. It consists on a learning strategy based on two successive stages which combines unsupervised learning. A self-organized learning scheme (i.e., unsupervised learning) is used to detect the potential fuzzy logic rules and to locate initial membership functions, then a supervised gradient-descent learning procedures is used to optimally adjust the parameters of the membership functions for desired outputs.

The result of the fusion module is a predictive map of potential risks. This map can be regarded as a decision model alert. We mean by alert, the ability to get ahead of an event in time, space, or both. Indeed, the map produced provides the evolution of a phenomenon in medium and long-term consequences for each pixel. This leads to the definition of preventive strategies and policies depending on potential risk seriousness.

\subsubsection{Results and validation}

The validation of the proposed approach regards its application on the "Hydric erosion" risk affecting southern Tunisian region. To delimitate this risk, a case study was conducted using a subset of HYPERION hyperspectral dataset. In situ data include a slope and a lithofaçies maps describing soil properties. Interpretation and risk assessment consists to fuse abundances maps with in situ data using the proposed neuro-fuzzy model. As such, CNT's (Tunisian Remote sensing Center) experts have defined a set of 42 fuzzy rules defining the degree of risk as a function of slope value, lithofaçies class and the proportion of some materials in each pixel. Laterally, we defined the form of membership functions using sigmoidal function which is legitimately chosen to model data variability (Cox, 1999). Learning neuro-fuzzy model has been developed on the basis of 568 pixels. This phase was used to calibrate the prediction model by adjusting the parameters of membership functions and refining the fuzzy rules base. Finally, neuro-fuzzy model generates the risk map shown by Figure 13.

In order to evaluate the results, the predicted risks were overlaid to the observed risks from 2000 to 2008 by the CNT experts. Performance on training and validation data are presented in Table 3 which indicates that about $87.54 \%$ of the training data which fell within the high category coincided with high category, about $85.53 \%$ of moderately high category coincided with moderately high category, $83.09 \%$ of moderate category coincided with moderate and $90.3 \%$ of the low category coincided with low. For the training data sets, correct classification was $(94.2 \%)$ and number of misclassified entries about $(5.8 \%)$. For the validation data sets (49\% of the training sets), the correct classification was $(97 \%)$ and number of misclassified entries about $(3 \%)$. Some others methods of interpretation used were evaluated independently in terms of prediction accuracy. Table 4 summarizes the measurements of efficiency and quality obtained from confusion matrices. 

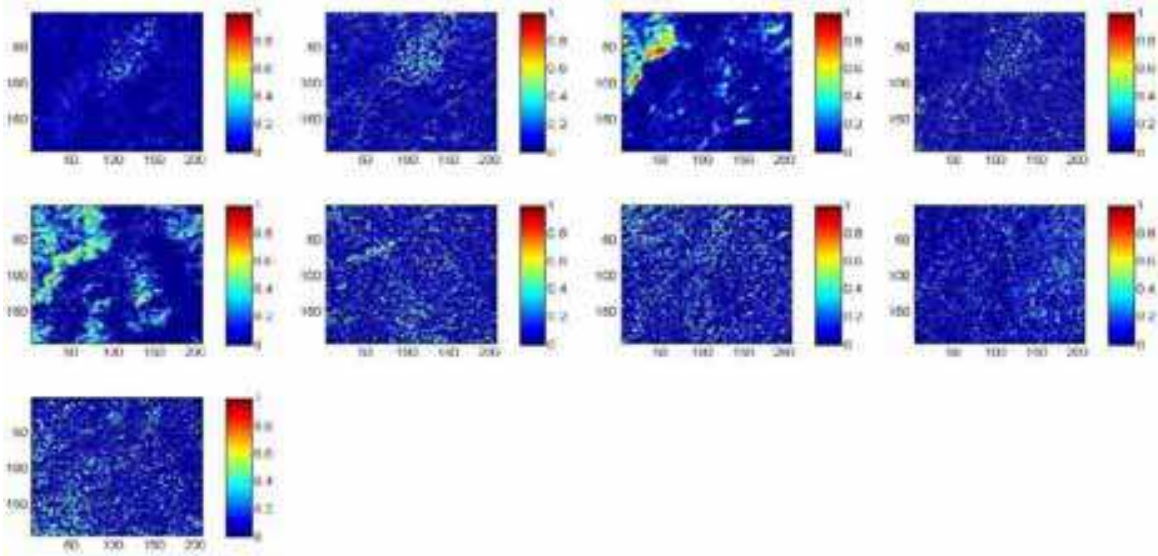

Fig. 12. Abundances maps,
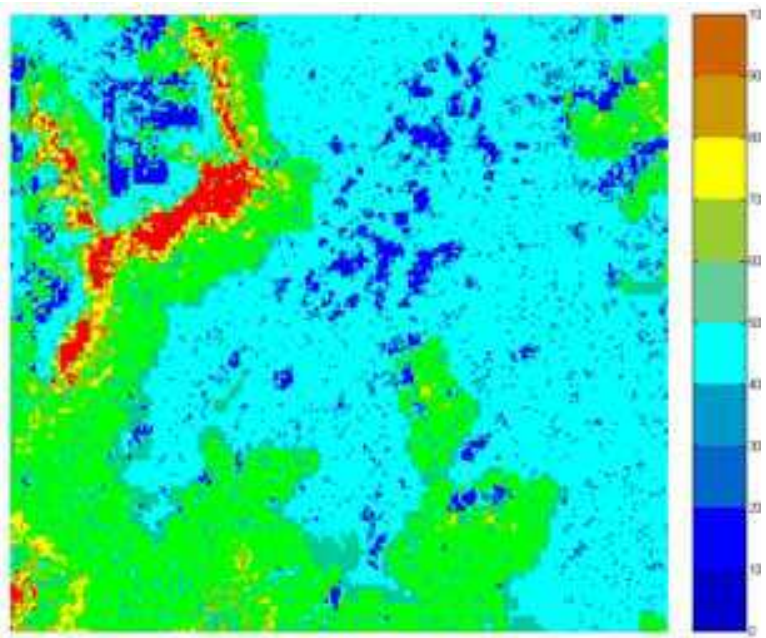

Fig. 13. Erosion risk map

\begin{tabular}{|c|c|c|c|c|}
\hline $\begin{array}{c}\text { Vulnerability } \\
\text { Classes }\end{array}$ & High & $\begin{array}{c}\text { Moderately } \\
\text { High }\end{array}$ & Moderate & Low \\
\hline High & 87.54 & 10.64 & 4.75 & 0.00 \\
\hline $\begin{array}{c}\text { Moderately } \\
\text { high }\end{array}$ & 9.77 & 85.53 & 8.94 & 3.27 \\
\hline Moderate & 2.15 & 3.00 & 83.09 & 6.43 \\
\hline Low & 0.54 & 0.83 & 3.22 & 90.3 \\
\hline
\end{tabular}

Table 3. Performance of the training data (\%) for erosion vulnerability

Table 4 allowed us to justify the choice of the neuro-fuzzy model. Indeed, comparing the average accuracy of the fuzzy approach $(75.04 \%)$ with neuronal prediction $(82.01 \%)$ and the maximum likehood $(83.49 \%)$, we can see the remarkable improvements $(91.9 \%)$ obtained by 
coupling these two techniques in one hybrid architecture. Furthermore, comparison of results obtained with the truth ground testifies the effectiveness of prevention diction of the approach proposed, this is expressed by a Kappa coefficient of about 0.7002 against 0.5847 for the fuzzy approach 0.6514 for neuronal prediction.

\begin{tabular}{|c|c|c|c|c|}
\hline $\begin{array}{c}\text { Vulnerability } \\
\text { Classes }\end{array}$ & $\begin{array}{c}\text { Neural } \\
\text { networks }\end{array}$ & $\begin{array}{c}\text { Fuzzy } \\
\text { logic }\end{array}$ & MLC & $\begin{array}{c}\text { Proposed } \\
\text { approach }\end{array}$ \\
\hline High & 87,32 & 87,35 & 89,17 & 90,07 \\
\hline $\begin{array}{c}\text { Moderately } \\
\text { high }\end{array}$ & 77,70 & 73.62 & 81.06 & 85.76 \\
\hline Moderate & 75.61 & 63.19 & 73.37 & 88.57 \\
\hline Low & 79,67 & 69.27 & 79.57 & 89.71 \\
\hline
\end{tabular}

Table 4. Comparison several approaches

\subsection{View 3: Towards a multi-approach image fusion 4.3.1 Introduction}

We have shown, in previous sections, that combining multi-sensor information provides a greater recognition accuracy and improves analysis quality. However, we have also noticed that satellite images interpretation is frequently marked by several types of imperfection. To overcome these weaknesses, most commonly approaches are probability, possibility, and evidence theories. Frequently, the major matter arising most studies is the choice of the most appropriate method for a particular situation and application issue. This section aims to present a novel approach consolidating several fusion techniques in order to choose the most appropriate depending on application field. By choosing the optimum theory for a particular image context, our approach will lead to improve images classification. Developed Framework is performed in the pixel level and it is based on a multi-agent system and a case-based reasoning.

\subsubsection{Proposed approach}

Data as available for an interpretation system are always somehow imperfect. Hence, imperfection, be it imprecision, uncertainty or ignorance, affect strongly most remotely sensed data and must be incorporated into every interpretation process. The term "imperfection" is usually used as a most general label. Materially, it can be due to imprecision, inconsistency, ignorance, uncertainty, etc (Farah et al., 2008b). Imprecision arises from the existence of a value, which cannot be measured with suitable precision. These imprecision can be resulting from a noise affecting satellite images that should be treated by applying some filters. Uncertainty is a property that arises from a lack of information about application nature. The uncertainty is resulting from an unreliable sensor or from spatial or temporal constraints. Imprecision and inconsistency are essentially properties of the information itself whereas uncertainty is a property of the relation between the information and our knowledge about context. The incompleteness reflects the fact that information is unable to capture all relevant aspects of an observable event (Bloch, 1996).

Conventionally, data imperfection was fluently modeled by probability theory. Until recently, many new theories have been proposed to deal with this problem. The large number of theories reflects the recent acknowledgement that probability theory, as good as it is, is not the unique alternative and it is not able to take into account all aspects of data 
imperfection (Mahler, 2007). Then, the use of inappropriate, unjustified, or purely one theory can lead to decline interpretation task and results. Moreover, the majority of interpretation systems do not hold into account the imperfection accompanying satellite images. Few systems use only one theory with very restricted parameters.

In this view, in order to handle data imperfection, we propose a new intelligent multiapproach for uncertain satellite images fusion combining three different data fusion methods, namely, the probability, possibility, and evidence methods. This system can provide a powerful framework for multi-sensor images fusion and decision-making. Therefore, the proposed architecture incorporates the information extracted by learning. It includes also some "structures detection" modules based on a set of agents; each of them is specialized in the detection of a specific object type.

Figure 14 summarized the proposed approach, which enclosed three levels of abstraction: the low level, the intermediate level and the high level. The three levels are independent and cooperate to build the whole image fusion and interpretation process. As shown, proposed approach is based on a multi-agent architecture. Interest for multi-agent approach is motivated by many factors (Tupin et al., 1999). Primary, as the fusion cell can be decomposed into several well-defined stages; each will be accountable of an independent processing agent. Second, agent's interaction, communication and cooperation induce a robust treatment process, allowing us to solve difficult situations and to reduce imperfection rate (Farah et al., 2006). Thus, a high performance of application can be achieved through parallelism between agents. Agents for each level communicate with their counterparts at other levels in order to answer requests and to transmit respective information. In our system, agent's knowledge will be stored in the fact basis, allowing a subsequent reasoning step using a set of rules. The learning process is necessary to initialize the multi-approach and images fusion. The agent of each abstraction level carries on and cooperates and generates information to the upper level in order to achieve interpretation task.

\subsubsection{Low-level abstraction:}

This level assures the extraction of symbolic information such as borders or homogeneous regions. Adopted techniques are intensely associated with data type, but they are independent of the application domain. In our approach, we choose to develop a set of agents allowing the extraction of useful information for interpretation and fusion tasks such as the learning agent, the structure detection agent (river detection, urban detection, etc.), the probability agent, the possibility agent, and the evidence agent. To better monitor imperfections, the process initializing our system emphasizes a learning process. Learning can be supervised or unsupervised allowing functions estimation.

\subsubsection{Intermediate-level abstraction:}

The intermediate level performs the designation of symbolic primitives extracted in previous level. This level is more sensitive and expresses a notable importance sense it provides an articulation component between low and high levels.

Depending on application's field, this level can be decomposed into several sub-levels; each of them is designed for specific kind of primitives and achieving to a particular transformation or a selection. In our case, we develop three types of intermediate-level agents, namely, the supervisor structure detection agent, the supervisor fusion agent, and the supervisor learning agent. The information gathered by the low-level agents is sent to the supervisor detection agent who must use knowledge about this information offered by the high level Decision 
support system (DSS). The DSS allows recognizing the type of the extracted zones through a set of rules stored in the rule basis. The rules are expressed in a language that is close to natural language, allowing DSS enrichment. We develop three types of rules:

1. Radiometric rules: modeling the shade level.

2. Geometric rules: concerning pixels arrangement.

3. Topologic rules: concerning the spatial relation and the position of the objects to detect. These three kinds of rules do not have the same weight to all images objects. For example, to validate the presence of an urban zone, the geometric criterion is more relevant than the radiometric and the topologic one. Moreover, the structures' description is often imprecise. To detect structures from images, we develop a set of agents, each of them designed to detect a specific object type. We can find for example the humid detection agent, the river detection agent, the urban detection agent, and the road detection agent.

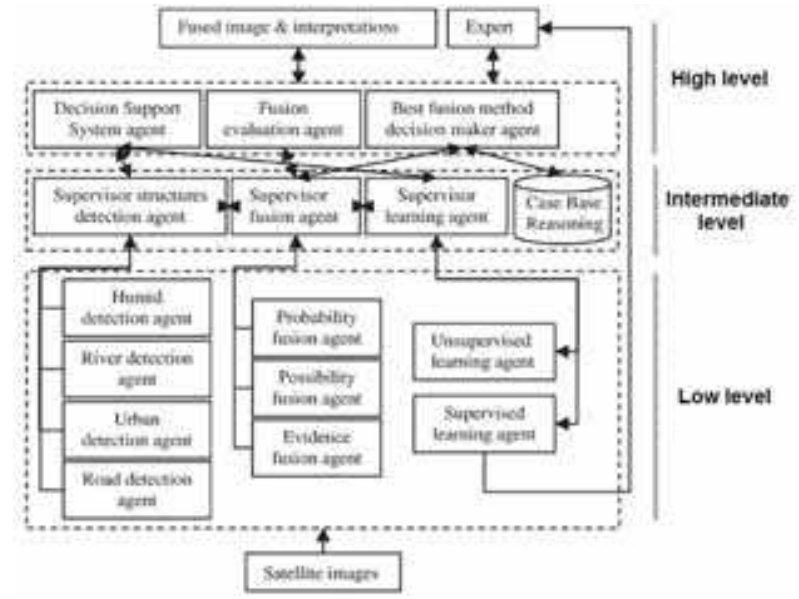

Fig. 14. Workflow of proposed multi-approach

\subsubsection{High-level abstraction:}

This level incorporates the interpretation mechanisms and symbolic representation of the scene. Information provided by learning and structure detection agents of intermediate level are used by the high-level fusion agents in order to build the resulting fused image.

In order to optimize the interpretation process, we have developed an agent called the best fusion method decision maker. This agent refers to case-based reasoning (CBR) module, which is particularly useful for applications where we lack sufficient knowledge either for formal representation or for parameter estimation (Bentabet et al., 2002). CBR presents cases related to similar previously handled problems; it suggests the solution adapted under similar situations and decides what order previous cases can provide for dealing with the current problem.

This module stores an archive about different fusion cases previously handled (Jurisica \& Glasgow, 2004). In our approach, each case has three components:

- The features describing each case: including textual, shape, color, and texture features;

- Image fusion method: gives a solution to a given problem

- The case relevance: provided by an expert.

For a better characterization of problem, we have weighed each problem feature according to its importance. A communication is launched between the supervisor fusion agent and 
the best fusion method decision-maker. For each image, we start by determining the features described above. Then we retrieve the best fusion method using a quadtree technique, allowing a multilevel structure representation of image features. Each level contains a set of nodes reserved for a specific feature (textual, color, texture, or shape).

The quadtree technique lets us to filter images by gradually increasing the detail level (Inglada \& Mercier, 2007). Image retrieval can be done in two ways. Globally by comparing globally the query image with all case base images, or using a region-based image retrieval in which each image in the database is split into different regions by the fuzzy c-means method (Archambeau et al. , 2006); then, each region in the input image is compared with all regions in the image in the basis.

For similarity measurement, the Bhattacharyya distance has been adopted (Deb \& Zhang, 2004). The distance between two images is computed using the distance between most resembling couples, excluding those having a distance less than a given threshold th.

After retrieving the closest image to the input one, the fusion method is deduced from the corresponding case. If the case basis does not contain a case similar to the current one, the three low-level fusion agents are launched.

The last step consists of evaluating the fusion method. The goal of the evaluation agent is to help the expert select the best fusion method for a given sequence of images. In order to accomplish that, we opted for a post-fusion analysis based on a confusion matrix.

\subsubsection{Results and validation}

In order to evaluate our multi-approcha fusion, we have used data presented in view 1 . The possibility method with the T-norm operator was selected as the most suitable fusion method for the first example. For the second example, the possibility method with the mean operator is the most suitable. Tables 5 and 6 shows, respectively, confusion matrices for the possibility methods (T-norm and mean operators).

To evaluate the performance of the proposed approach, we compare CBR results with those obtained following methods: the probability method established on equiprobablity between the five images, the possibility method applied with three types of combination operators (Tnorm, T-conorm, and mean). The last method is an unsupervised fusion by evidence theory. The images resulting from these fusion methods will be compared according to OK criteria.

\begin{tabular}{|l|l|l|l|l|l|}
\hline & 1 & 2 & 3 & 4 & 5 \\
\hline 1 & 98.33 & 1.5 & 0.17 & 0.00 & 0.12 \\
\hline 2 & 1.55 & 94.75 & 3.32 & 0.38 & 2.88 \\
\hline 3 & 0.11 & 3.59 & 91.05 & 4.92 & 1.28 \\
\hline 4 & 0.01 & 0.16 & 5.33 & 92.68 & 2.06 \\
\hline 5 & 0.00 & 0.00 & 0.13 & 2.02 & 92.66 \\
\hline
\end{tabular}

Table 5. Confusion matrix of the first example.

\begin{tabular}{|c|c|c|c|c|c|}
\hline & 1 & 2 & 3 & 4 & 5 \\
\hline 1 & 93.11 & 1.03 & 3.73 & 2.01 & 0.12 \\
\hline 2 & 1.02 & 93.16 & 2.07 & 0.87 & 2.88 \\
\hline 3 & 3.75 & 1.91 & 89.05 & 4.01 & 1.28 \\
\hline 4 & 2.01 & 0.82 & 2.22 & 92.89 & 2.06 \\
\hline 5 & 0.11 & 3.08 & 2.93 & 0.22 & 92.66 \\
\hline
\end{tabular}

Table 6. Confused matrix of the second example. (1:Humid, 2:Parcel, 3:Cultivated, 4:Urban, 5:Sebkha) 
Tables 7 and 8 present a comparison between the probability, possibility, and evidence fusion methods for examples 1 and 2, respectively, according to OK criteria.

As we can see, the possibility method with T-norm and mean operators has the best value for the assessment criteria of the two examples. This result, in perfect correspondence with CBR one, proves that our approach seems to be useful and effective. It allows interpretation process optimization by avoiding the call of the three fusion methods.

\begin{tabular}{|c|c|}
\hline Assessment parameter & OK \\
\hline Probability theory & 0.891 \\
\hline Possibilty theory (1) & 0.932 \\
\hline Possibilty theory (2) & 0.748 \\
\hline Possibilty theory (3) & 0.889 \\
\hline Evidence theory & 0.921 \\
\hline
\end{tabular}

Table 7. Evaluation of three fusion method for the fist example.

\begin{tabular}{|c|c|}
\hline Assessment parameter & OK \\
\hline Probability theory & 0.882 \\
\hline Possibilty theory (1) & 0.781 \\
\hline Possibilty theory (2) & 0.866 \\
\hline Possibilty theory (3) & 0.893 \\
\hline Evidence theory & 0.849 \\
\hline
\end{tabular}

Table 8. Evaluation of three fusion method for the second example.

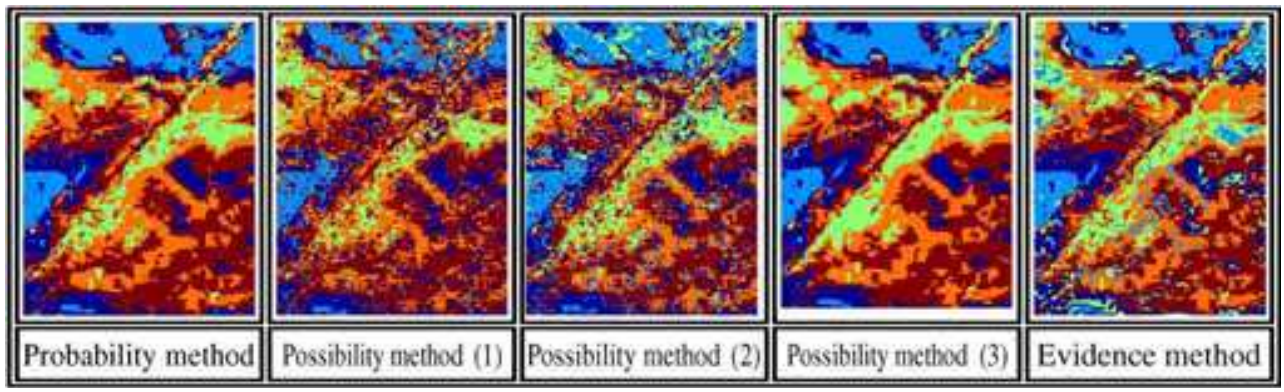

Fig. 15. Classified images for the second example.

\section{Discussion}

In this chapter, we have presented several views for satellite images fusion. As shown, several kinds of problems hamper and dampen the quality of a reliable images fusion. Images fusion, especially multi-sensor one, is limited by several factors. First, simultaneously acquired multisensor images are not always available for the same area and time. Moreover, interpretation is usually limited by spatial resolutions unconformity and data incompatibility. Since there is no common recognized procedure to do this, most studies are regularly forced to find empirically the best fusion scheme, the most useful data and optimal results. Numerous authors have agreed that the fusion should be done usually from separate and heterogeneous data sources. We have introduced, in this chapter, a different kind of fusion that is made at the sub-pixel 
level. Thus, the transformation performed at each pixel attempt to provide additional fusion sources. This investigation leads us to overcome the assumption of pixels homogeneity in remotely sensed data. Therefore, newest sources, extracted by bind source separation, offers a new precise and detailed knowledge about land cover proprieties.

We have also addressed the dilemma related to an efficient combination of symbolic knowledge into fusion process in order to increase interpretation quality. We have thus proposed an appropriate framework for this fusion allowing an efficient fusion of both images (extracted additional sources) and In-situ data modelled by a fuzzy rule basis. A learning capability has been added to calibrate and adjust the model.

We also showed that the choice of a specific theory for fusion is a tedious task which must be done carefully; taking into account several parameters such as study context, available data etc. Proposed multi-view is an accomplished way for finest interpretation of large volumes data from multiple sources. Against feature or decision level fusion, all proposed views in this chapter operate in the pixel and sub-pixel have the opportunity to use all available original data. This leads us to reduce the loss of information occurring during the feature extraction process.

\section{Conclusions}

In this chapter, we reviewed some images fusion approaches on remote sensing field. We have shown, by exposing various interpretation views, that the sub-pixel fusion level has become a successful way to overcome difficulties related to multi-sensor and multi-source images fusion. Hence, the growth of signal processing techniques and symbolic knowledge enable a new fusion leading to an enhanced interpretation quality. Besides knowledge integration, the election of the optimum fusion approach and results evaluation of pixel and sub-pixel level image fusion have been well studied in this chapter, each view was designed to solve a specific fusion issue.

Obtained results show that the sub-pixel fusion level has been rapidly developing and gradually becoming mature. Therefore, fusion process issues and practical matters associated with the implementation of such image fusion strategy should be considered seriously. Challenges remain with regard to developing intelligent fusion methods adapting to vastly different situations. However, there still remain many issues that deserve to be studied further such as mathematic formulation and learning incorporation. In addition, the development of the sub-pixel level image fusion techniques urgently demands widely accepted, objective quality metrics.

\section{References}

Archambeau, C.; Valle, M.; Assenza, A. \& Verleysen M. (2006). Assessment of probability density estimation methods: Parzen window and finite Gaussian mixtures, In proceedings of IEEE ISCAS, Vol.11, No.2, pp. 3245-3248, ISBN: 0-7803-9389-9, Septembre 2006.

Babaie-Zadeh, M.; \& Jutten, C. (2005). A general approach for mutual information minimization and its application to blind source separation. Signal Processing, Vol. 85, No.5, (May 2005) (975-995), ISSN:0165-1684.

Belouchrani, A.; Abed-Meraim, K.; Cardoso J.-F.; \& Moulines, E. (1997). A blind source separation technique using second-order statistics. IEEE Transactions on signal processing, Vol. 45, No.2, (February 1997) (434-444), ISSN:1053-587X. 
Bentabet, L.; Jodouin, S.; \& Boudraa, A. (2002). Iterative estimation of Dempster-Shafer's basic probability assignment: application to multisensor image segmentation. Optical Engineering, Vol. 41, No.4 , (April 2004)(760-770), ISSN:0091-3286.

Bloch, I. (1996). Information Combination Operators for Data Fusion : A Comparative Review with Classification. IEEE Transactions on Man, and Cybernetics - Part A : Systems and Humans, Vol. 26, No.1 , (Janvier 1996)( 52-67), ISSN:0196-2892.

Cao, X.R. \& Liu, R.-W. (1996). General approach to blind source separation. IEEE Transactions on signal processing, Vol. 44, No. , (1996) (562-571), ISSN: 1053-587X.

Cardoso, J.-F.; \& Souloumiac, A. (1993). Blind beamforming for non Gaussian signals, IEEE proceedings of Radar and Signal Processing, Vol. 140, No.6, pp. 362 - 370, 0956-375X, Toulouse France, December 1993.

Chang, C.-I., \& DU, Q. (2004). Estimation of the number of spectrally distinct signal sources in hyperspectral imagery. IEEE Transactions on Geoscience and Remote Sensing, Vol. 44, No.3 , (2004)(608-619), ISSN 0196-2892.

Cox, E. (1994). The fuzzy systems handbook: a practitioner's guide to building, using, and maintaining fuzzy systems, Academic Press Professional, ISBN:0-12-194270-8, San Diego, CA, USA.

Dasarathy, B.V. (2001). Information fusion - what, where, why, when, and how? Information Fusion, Vol. 6, No.4, (December 2005)( 75-76).

Deb, S. \& Zhang, Y. (2004). An overview of content-based image retrieval techniques, In proceedings of 18th Int. Conf. AINA, Vol., No., pp. 59-64, 2004.

Farah, I.R; \& Ahmed, M. B. (2010). Towards an intelligent multi-sensor satellite image analysis based on blind source separation using multi-source image fusion. IJRS International Journal of Remote Sensing : Taylor \& Francis Vol. 31, No. 1, (10 January 2010)(13-38).

Farah(a), I.R; Boulila, W.; Saheb Ettabaâ, K.; Solaiman, B.; Ben Ahmed, M. (2008). Interpretation of multisensor remote sensing images: Multi-approach fusion of uncertain information. IEEE Transactions on Geoscience and Remote Sensing, Vol. 46, No.12 , (Decembre)(4142-4152), ISSN 0196-2892.

Farah(b), I.R; Boulila, W.; Saheb Ettabaâ, K.; Solaiman, B.; Ben Ahmed, M. (2008). Multiapproach system based on fusion of multi-spectral image for land cover classification. IEEE Transactions on Geoscience and Remote Sensing, Vol. 46, No.12 , (Decembre)( 4153-4161), ISSN 0196-2892.

Farah, I.R.; Saheb Ettabaa, K. \& Ben Ahmed M. (2006). A generic multi-agent system for analyzing spatial-temporal geographic information. International Journal of Computer Science and Network Security, Vol. 6, No.8 , (Aug 2006)(4-10).

Farah, I.R.; Ahmed, M.B.; \& Boussema, M.R. (2003). Multispectral satellite image analysis based on the method of blind separation and fusion of sources, Proceedings of IEEE International Geoscience and Remote Sensing Symposium, pp. 3638-3640, ISBN, Toulouse France, May 2003.

Gamba, P.; Dell'Acqua, F.; V. Dasarathy, B.V. (2005). Urban remote sensing using multiple data sets: Past, present, and future. Information Fusion, Vol. 6, No.4 , (December 2005)(319-326).

Gianinetto, M. \& Villa, P. (2007). Rapid response flood assessment using minimum noise fraction and composed spline interpolation. IEEE Transactions on Geoscience and Remote Sensing, Vol. 45, No.10, (Octobre 2007)( 3204-3211), ISSN: 0196-2892.

Goshtasby, A. (2005). 2-D and 3-D Image Registration for Medical, Remote Sensing, and Industrial Applications, Wiley Publishers, ISBN: 0123725291. 
Hall Dave L. \& Llinas, J. (1997). Introduction to Multisensor Data Fusion, Proc. of IEEE, Vol.85, No.1, pp. 6 - 23, ISSN: 0018-9219, January 1997.

Hemissi, S.; Ben Rabah Z. B. ; Farah, I.R ; Mercier, G. \& Solaiman B. (2009). Un modèle neuro-flou pour l'interprétation d'images hyperspectrales : application à la gestion des risques, TAIMA 2009 Traitement et Analyse de l'Information : Méthodes et Applications, May 2009, Hammamet Tunisia.

Hyvärinen, A. \& Oja, O. (1997). A Fast Fixed-Point Algorithm for Independent Component Analysis. Neural Computation, Vol. 9, No., (1997) (1483--1492), ISSN:0899-7667.

Inglada, J. \& Mercier, G. (2007). A new statistical similarity measure for change detection in multitemporal SAR images and its extension to multiscale change analysis. IEEE Transactions on Geoscience and Remote Sensing, Vol. 45, No.5, (May 2007)(1432-1445), ISSN:0196-2892.

Jurisica, I. \& Glasgow, J. (2004). Applications of case-based reasoning in molecular biology. Artificial Intelligence Magazine. - Special Issue on Bioinformatics, Vol. 25, No.1 , (May 2004)(85-95), ISSN:0738-4602.

Lawrence A. Klein (2004). Sensor and Data Fusion: A Tool for Information Assessment and Decision Making (SPIE Press Monograph Vol. PM138), SPIE- International Society for Optical Engineering, ISBN: 0819454354.

Lin, C.J.; \& Lin, C.T. (1997). An ART-based fuzzy adaptive learning control network. IEEE Transactions on Fuzzy Systems, Vol. 5, No.4 , (Novembre 1997)(477 - 496), ISSN:10636706.

Mahler Ronald P. S. (2007). Statistical Multisource-Multi-target Information Fusion, Artech House Inc, ISBN:9781596930926, Norwood MA USA.

Mansour, A.; Barros, A.K; \& Ohnish, N. (2000). Blind separation of sources: methods, assumptions and applications. IEICE Transactions on Fundamentals of Electronics, Communications and Computer Sciences, Special Section on Digital Signal Processing, Vol. 83, No.A , (2000)(1498-1512).

Pohl, C.; Van Genderen, J.L. (1998). Review article: multisensor image fusion. In Remote sensing: concepts, methods and applications. International Journal of Remote Sensing, Vol. 19, No.5, ()(823-854).

Stathaki, T. (2008). Image Fusion: Algorithms and Applications, Academic Press, ISBN: 0123725291.

Tupin, F.; Bloch, I.; \& Maître. H. (1999). A first step toward automatic interpretation of SAR images using evidential fusion of several structure detectors. IEEE Transactions on Geoscience and Remote Sensing, Vol. 27, No.3 , (May 1999)( 1327-1343), ISSN: 0196-2892.

Wald, L. (1999). Some terms of reference in data fusion. IEEE Transactions on Geoscience and Remote Sensing, Vol. 37, No.3 , (May 1999) (1190 - 1193), 0196-2892, ISSN: 0196-2892.

Wang, J.; \& Chang, C.-I. (2006). Applications of independent component analysis in endmember extraction and abundance quantication for hyperspectral imagery. IEEE Transactions on Geoscience and Remote Sensing, Vol. 44, No.9 , (September 2006)( 2601 - 2616), 0196-2892, ISSN: 0196-2892.

Yuhas, R.H.; Goetz, A.F.H.; \& Boardman, J.W. (1992). Discrimination Among Semi-Arid Landscape Endmembers Using the Spectral Angle Mapper (SAM) Algorithm, Summaries of the 4th JPL Airborne Earth Science Workshop, Vol. 92, No.41, pp. 147-149.

Yu, H.-L.; Christakos, G. (2010). Modeling and Estimation of Heterogeneous Spatiotemporal Attributes Under Conditions of Uncertainty. IEEE Transactions on Geoscience and Remote Sensing, Vol. 48, No. 9, (Aout 2010) 11 (1 - 11). 


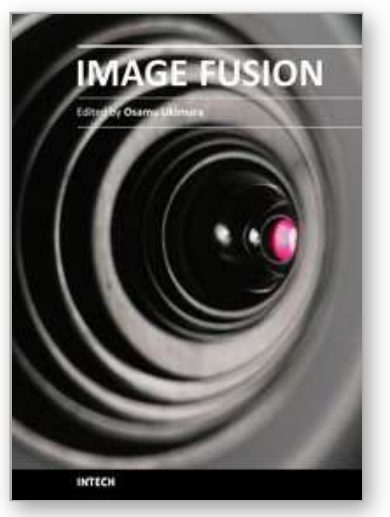

\author{
Image Fusion \\ Edited by Osamu Ukimura
}

ISBN 978-953-307-679-9

Hard cover, 428 pages

Publisher InTech

Published online 12, January, 2011

Published in print edition January, 2011

Image fusion technology has successfully contributed to various fields such as medical diagnosis and navigation, surveillance systems, remote sensing, digital cameras, military applications, computer vision, etc. Image fusion aims to generate a fused single image which contains more precise reliable visualization of the objects than any source image of them. This book presents various recent advances in research and development in the field of image fusion. It has been created through the diligence and creativity of some of the most accomplished experts in various fields.

\title{
How to reference
}

In order to correctly reference this scholarly work, feel free to copy and paste the following:

Farah Imed Riadh (2011). A Multi Views Approach for Remote Sensing Fusion Based on Spectral, Spatial and Temporal Information, Image Fusion, Osamu Ukimura (Ed.), ISBN: 978-953-307-679-9, InTech, Available from: http://www.intechopen.com/books/image-fusion/a-multi-views-approach-for-remote-sensing-fusionbased-on-spectral-spatial-and-temporal-information

\section{INTECH}

open science | open minds

\section{InTech Europe}

University Campus STeP Ri

Slavka Krautzeka 83/A

51000 Rijeka, Croatia

Phone: +385 (51) 770447

Fax: +385 (51) 686166

www.intechopen.com

\section{InTech China}

Unit 405, Office Block, Hotel Equatorial Shanghai

No.65, Yan An Road (West), Shanghai, 200040, China

中国上海市延安西路65号上海国际贵都大饭店办公楼405单元

Phone: +86-21-62489820

Fax: +86-21-62489821 
(C) 2011 The Author(s). Licensee IntechOpen. This chapter is distributed under the terms of the Creative Commons Attribution-NonCommercialShareAlike-3.0 License, which permits use, distribution and reproduction for non-commercial purposes, provided the original is properly cited and derivative works building on this content are distributed under the same license. 